Supplement of Clim. Past Discuss., 11, 637-670, 2015

http://www.clim-past-discuss.net/11/637/2015/

doi:10.5194/cpd-11-637-2015-supplement

(C) Author(s) 2015. CC Attribution 3.0 License.

(c) (i)

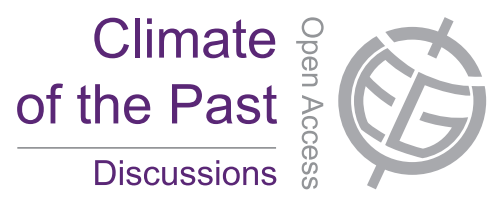

Supplement of

\title{
Trace metal evidence for a poorly ventilated glacial Southern Ocean
}

M. Wagner and I. L. Hendy

Correspondence to: M. Wagner (wagne2me@cmich.edu) 


\section{Supplement}

\section{$2 \quad$ S1. ${ }^{230}$ Th Normalization}

Lateral sediment redistribution is a well-documented phenomenon in the Southern Ocean

4 that can deposit up to 20 times more sediment than the vertical rain at a given site (Chase et al.,

5 2003; Dezileau et al., 2000; Francois et al., 1993). To correct for this, prior studies have

6 normalized proxy concentrations to ${ }^{230} \mathrm{Th}$ (e.g., Anderson et al., 2009; Bradtmiller et al., 2009;

7 Chase et al., 2003), which also generates fluxes of proxies to the sediment-water interface

8 (Francois et al., 2004). Thorium-230 has a known production rate in the water column, and its

9 expected flux to sediments is entirely a function of water depth. Preserved vertical fluxes of

10 sedimentary components are calculated from measured excess ${ }^{230} \mathrm{Th}$ and normalized to the

11 expected flux of ${ }^{230}$ Th to sediments (Francois et al., 2004):

$$
F_{i}=\left(\beta \times z \times f_{i}\right) /{ }_{\mathrm{xs}}^{230} \mathrm{Th}_{0}
$$

13 where $F_{i}$ is the preserved vertical flux of component $i ; \beta$ is the production rate of ${ }^{230} \mathrm{Th}$ in the

14 water column $\left(2.63 \times 10^{-5} \mathrm{dpm} / \mathrm{cm}^{3} / \mathrm{kyr}\right) ; z$ is water depth in $\mathrm{cm} ; f_{i}$ is the weight fraction of

15 component $i$; and ${ }_{\mathrm{xs}}^{230} \mathrm{Th}_{0}$ is the measured sedimentary ${ }^{230} \mathrm{Th}$ not associated with the detrital

16 component.

\section{S2. Trace Metal Fluxes}

18 To assess whether interpretation of redox chemistry may have been affected by sediment

19 redistribution, trace metal and organic carbon concentrations (Tables S1-S4) were normalized to

$20{ }^{230} \mathrm{Th}$. Published ${ }_{\mathrm{xs}}^{230} \mathrm{Th}_{0}$ data (Anderson et al., 2009, archived data; Kumar et al., 1995, Table

21 S5) and modern water depths for cores were used to calculate preserved vertical fluxes to

22 sediments. ${ }_{x s}^{230} \mathrm{Th}_{0}$ values were linearly interpolated between data points, except for sampled

23 intervals at the tops of the cores above the first data point, which were assigned the same ${ }_{\mathrm{xs}}^{230} \mathrm{Th}_{0}$ 
24 value as the first data point; and sampled intervals below the last data point $(884 \mathrm{~cm})$ in TN057-

25 13-4PC, which were assigned the same ${ }_{\mathrm{xs}}{ }^{230} \mathrm{Th}_{0}$ value as the last data point. Errors for

26 interpolated ${ }_{\mathrm{xs}}^{230} \mathrm{Th}_{0}$ values were estimated by calculating one standard deviation of the ${ }_{\mathrm{xs}}{ }^{230} \mathrm{Th}_{0}$

27 values immediately above and below the sampled interval. Errors in ${ }_{\mathrm{xs}}{ }^{230} \mathrm{Th}_{0}$ and trace metal

28 concentrations were propagated at the $1 \sigma$ level when calculating trace metal fluxes.

29 Given that trace metals precipitate at a redox front within sediments, and ${ }^{230} \mathrm{Th}$ is

30 delivered to the sediment-water interface in association with particles, a vertical offset between

31 trace metals and ${ }^{230}$ Th can occur. However, this offset is likely minimal (see Section 2.1) and

32 less important given large other uncertainties present in calculating trace metal fluxes (discussed

33 below).

34 Total uncertainty in trace metal fluxes arises mostly from low data density for ${ }_{\mathrm{xs}}{ }^{230} \mathrm{Th}_{0}$

35 values in RC13-254, and the choice to linearly interpolate between data points for both cores.

36 Without additional data points, the true variation in $\mathrm{RC} 13-254{ }_{\mathrm{xs}}{ }^{230} \mathrm{Th}_{0}$ values is unknown. In

37 comparison, uncertainties in trace metal fluxes for TN057-13-4PC are much smaller and of less

38 concern, particularly during deglaciation, and they will not be considered further. For RC13-254,

39 large uncertainties in trace metal fluxes make it largely impossible to discern variation through

40 time, with the exception of $\sim 140 \mathrm{~cm}(\sim 17.7 \mathrm{ka})$. However, we can consider two extreme cases:

41 that the true trace metal fluxes are either at the upper or lower bounds of the uncertainty window.

42 If fluxes are at the upper bound, this strongly indicates reducing conditions in sedimentary pore

43 waters from high export productivity and/or low bottom water oxygen prior to $\sim 13.5$ ka (below

$44 \sim 65 \mathrm{~cm}$ core depth). If fluxes are at the lower bound, there is a smaller-but discernible-

45 difference between the intervals of $\sim 3.5-15.6 \mathrm{ka}(0-102 \mathrm{~cm}$; burndown interval) and $\sim 15.6-29 \mathrm{ka}$

$46(102-380 \mathrm{~cm})$ [Figure S1], indicating a change to better oxygenated conditions sometime after 
$47 \sim 15.6 \mathrm{ka}$. Trace metal fluxes (Figures S1 and S2) are, however, consistent with total bulk 48 sediment trace metal concentrations (Figures 3 and 4) in that they demonstrate higher input to 49 sediments during the last glacial period and deglaciation. Furthermore, correspondence with $50 \Delta \Delta^{14} \mathrm{C}$ ventilation records (Skinner et al., 2010; see Section 4.3; Skinner et al., 2014) and organic 51 carbon concentrations/fluxes (Figures 3 and 4; Figures S1 and S2) argues against trace metal 52 accumulation being an artifact of sediment focusing.

53 One important difference between trace metal fluxes and concentrations arises at site 54 TN057-13-4PC from $\sim 17 \mathrm{ka}(\sim 770 \mathrm{~cm})$ onward. Normalization to ${ }^{230} \mathrm{Th}$ produces several peaks 55 in Ag and Re fluxes that do not otherwise appear or are muted when plotting total bulk sediment 56 trace metal concentrations. Elevated opal (Figure 4f) and organic carbon (Figure S2) fluxes 57 suggest that any trace metal enrichments are productivity driven. 


\section{Supplementary Figure Captions}

60

61 Figure S1. Trace metal and organic carbon fluxes for RC13-254.

62

63 Figure S2. Trace metal and organic carbon fluxes for TN057-13-4PC.

64 
65

66 Supplementary Tables

67

68 Table S1. TN057-13-4PC trace metal concentrations.

\begin{tabular}{|c|c|c|c|c|c|c|c|c|c|}
\hline Depth $(\mathrm{cm})$ & Age (yr) & $\mathrm{Cd}(\mathrm{ppm})$ & $\begin{array}{c} \pm 1 \sigma \\
(\mathrm{ppm})\end{array}$ & $\operatorname{Re}(p p b)$ & $\begin{array}{l} \pm 1 \sigma \\
(\mathrm{ppb})\end{array}$ & $\mathrm{Ag}(\mathrm{ppb})$ & $\begin{array}{l} \pm 1 \sigma \\
(\mathrm{ppb})\end{array}$ & Mo (ppm) & $\begin{array}{c} \pm 1 \sigma \\
(\mathrm{ppm})\end{array}$ \\
\hline $0-1$ & 18 & 0.06 & 0.01 & 0.5 & 0.0 & 69 & 15 & 0.46 & 0.10 \\
\hline $10-11$ & 305 & 0.10 & 0.02 & 0.4 & 0.0 & 19 & 3 & 1.29 & 0.22 \\
\hline $20-21$ & 749 & 0.03 & 0.01 & 0.4 & 0.0 & 45 & 6 & 0.18 & 0.00 \\
\hline $40-41$ & 1259 & 0.07 & 0.01 & 0.4 & 0.0 & 37 & 5 & 0.12 & 0.00 \\
\hline $60-61$ & 1769 & 0.08 & 0.02 & 0.5 & 0.0 & 70 & 10 & 0.08 & 0.00 \\
\hline $80-81$ & 2295 & 0.10 & 0.02 & 0.7 & 0.0 & 79 & 11 & 0.08 & 0.00 \\
\hline $100-101$ & 2824 & 0.10 & 0.02 & 0.6 & 0.0 & 74 & 10 & 0.14 & 0.00 \\
\hline $120-121$ & 3353 & 0.10 & 0.02 & 0.6 & 0.0 & 61 & 9 & 0.18 & 0.04 \\
\hline $140-141$ & 3882 & 0.12 & 0.02 & 1.0 & 0.0 & 71 & 10 & 0.10 & 0.00 \\
\hline $160-161$ & 4410 & 0.13 & 0.02 & 0.6 & 0.0 & 38 & 8 & 0.12 & 0.00 \\
\hline $180-181$ & 4939 & 0.14 & 0.03 & 1.0 & 0.0 & 63 & 9 & 0.12 & 0.00 \\
\hline $200-201$ & 5468 & 0.20 & 0.04 & 1.0 & 0.0 & 81 & 11 & 0.12 & 0.00 \\
\hline $220-221$ & 5997 & 0.26 & 0.05 & 2.4 & 0.1 & 142 & 20 & 0.16 & 0.00 \\
\hline $240-241$ & 6526 & 0.25 & 0.04 & 13.3 & 0.6 & 145 & 31 & 0.47 & 0.08 \\
\hline $260-261$ & 6998 & 0.24 & 0.04 & 9.9 & 0.5 & 87 & 12 & 0.45 & 0.01 \\
\hline $300-301$ & 7549 & 0.19 & 0.03 & 4.1 & 0.2 & 88 & 12 & 0.48 & 0.01 \\
\hline $340-341$ & 8100 & 0.20 & 0.04 & 3.6 & 0.2 & 125 & 18 & 0.53 & 0.01 \\
\hline $380-381$ & 8651 & 0.18 & 0.07 & 4.4 & 0.2 & 148 & 32 & 0.52 & 0.01 \\
\hline $420-421$ & 9203 & 0.17 & 0.03 & 1.9 & 0.1 & 74 & 10 & 0.39 & 0.01 \\
\hline $470-471$ & 9631 & 0.11 & 0.02 & 2.1 & 0.1 & 82 & 11 & 0.33 & 0.05 \\
\hline $510-511$ & 10164 & 0.10 & 0.02 & 2.7 & 0.1 & 84 & 18 & 0.39 & 0.01 \\
\hline $560-561$ & 10988 & 0.10 & 0.02 & 1.6 & 0.1 & 94 & 13 & 0.22 & 0.03 \\
\hline $580-581$ & 11367 & 0.09 & 0.02 & 0.8 & 0.0 & 76 & 11 & 0.30 & 0.00 \\
\hline $610-611$ & 11936 & 0.09 & 0.02 & 1.0 & 0.0 & 40 & 9 & 0.27 & 0.00 \\
\hline $640-641$ & 12505 & 0.08 & 0.02 & 1.5 & 0.1 & 45 & 6 & 0.26 & 0.00 \\
\hline $670-671$ & 13073 & 0.14 & 0.03 & 1.2 & 0.1 & 82 & 11 & 0.31 & 0.00 \\
\hline $700-701$ & 13642 & 0.15 & 0.03 & 2.7 & 0.1 & 81 & 11 & 0.71 & 0.01 \\
\hline $730-731$ & 14211 & 0.16 & 0.03 & 4.2 & 0.2 & 95 & 13 & 0.79 & 0.01 \\
\hline $760-761$ & 16163 & 0.13 & 0.02 & 9.6 & 0.5 & 93 & 13 & 0.86 & 0.14 \\
\hline $770-771$ & 17062 & 0.26 & 0.14 & 4.6 & 0.2 & 87 & 19 & 1.17 & 0.02 \\
\hline $780-781$ & 17960 & 0.34 & 0.06 & 5.0 & 0.2 & 130 & 18 & 1.31 & 0.23 \\
\hline $790-791$ & 18859 & 0.43 & 0.08 & 6.4 & 0.3 & 200 & 28 & 1.25 & 0.02 \\
\hline $800-801$ & 19758 & 0.42 & 0.07 & 13.0 & 0.6 & 216 & 47 & 1.38 & 0.23 \\
\hline $810-811$ & 20657 & 0.32 & 0.06 & 7.3 & 0.3 & 218 & 31 & 1.28 & 0.02 \\
\hline $820-821$ & 21556 & 0.23 & 0.04 & 3.7 & 0.2 & 71 & 15 & 1.03 & 0.18 \\
\hline $830-831$ & 22454 & 1.13 & 0.20 & 7.4 & 0.4 & 382 & 53 & 0.62 & 0.11 \\
\hline
\end{tabular}




\begin{tabular}{llllllllll}
$840-841$ & 23353 & 1.13 & 0.20 & 9.0 & 0.4 & 589 & 82 & 0.75 & 0.01 \\
$850-851$ & 24252 & 1.24 & 0.22 & 14.2 & 0.7 & 366 & 81 & 0.84 & 0.14 \\
$860-861$ & 25151 & 0.99 & 0.18 & 11.8 & 0.5 & 267 & 37 & 0.78 & 0.01 \\
$870-871$ & 26050 & 0.78 & 0.14 & 15.3 & 0.7 & 154 & 22 & 0.99 & 0.17 \\
$880-881$ & 26949 & 0.73 & 0.13 & 26.4 & 1.2 & 114 & 16 & 0.86 & 0.01 \\
$890-891$ & 27847 & 0.69 & 0.12 & 64.8 & 3.1 & 119 & 17 & 1.39 & 0.23 \\
$900-901$ & 28746 & 0.60 & 0.11 & 41.2 & 2.0 & 98 & 21 & 1.67 & 0.28 \\
$910-911$ & 29645 & 0.52 & 0.09 & 55.1 & 2.6 & 121 & 17 & 1.50 & 0.26 \\
$920-911$ & 30604 & 0.41 & 0.07 & 15.6 & 0.8 & 139 & 19 & 1.14 & 0.19 \\
\hline
\end{tabular}

69

70 
Table S2. RC13-254 trace metal concentrations.

\begin{tabular}{|c|c|c|c|c|c|c|c|c|c|}
\hline Depth $(\mathrm{cm})$ & Age (yr) & $\mathrm{Cd}(\mathrm{ppm})$ & $\begin{array}{c} \pm 1 \sigma \\
(\mathrm{ppm})\end{array}$ & $\operatorname{Re}(p p b)$ & $\begin{array}{l} \pm 1 \sigma \\
(\mathrm{ppb})\end{array}$ & $\mathrm{Ag}(\mathrm{ppb})$ & $\begin{array}{l} \pm 1 \sigma \\
(\mathrm{ppb})\end{array}$ & $\begin{array}{c}\text { Mo } \\
\text { (ppm) }\end{array}$ & $\begin{array}{c} \pm 1 \sigma \\
(\mathrm{ppm})\end{array}$ \\
\hline $0-1$ & 3500 & 0.87 & 0.15 & 1.3 & 0.1 & 18 & 3 & 1.19 & 0.46 \\
\hline $5-6$ & 4625 & $8.97 *$ & 1.56 & 0.8 & 0.0 & 11 & 2 & 0.21 & 0.04 \\
\hline $10-11$ & 5750 & 1.80 & 0.30 & 0.2 & 0.0 & 14 & 3 & 0.38 & 0.01 \\
\hline $15-16$ & 6950 & 0.13 & 0.02 & 0.5 & 0.0 & 4 & 1 & 0.57 & 0.01 \\
\hline $20-21$ & 8293 & $3.70^{*}$ & 0.74 & 0.5 & 0.0 & 24 & 5 & 0.70 & 0.14 \\
\hline $25-26$ & 9100 & 0.10 & 0.02 & 0.4 & 0.0 & 7 & 1 & 0.50 & 0.10 \\
\hline $30-31$ & 10100 & 0.07 & 0.01 & 0.6 & 0.0 & 1 & 0 & 0.91 & 0.01 \\
\hline $35-36$ & 11050 & 0.08 & 0.01 & 0.6 & 0.0 & 14 & 2 & 0.76 & 0.01 \\
\hline $41-42$ & 11650 & 0.01 & 0.00 & 0.7 & 0.0 & 24 & 3 & 0.35 & 0.01 \\
\hline $50-51$ & 12393 & 0.00 & 0.00 & 0.8 & 0.0 & 20 & 3 & 0.28 & 0.00 \\
\hline $61-62$ & 13125 & 0.02 & 0.00 & 1.0 & 0.0 & 31 & 4 & 0.51 & 0.01 \\
\hline $70-71$ & 13575 & 0.04 & 0.01 & 5.6 & 0.3 & 90 & 13 & 0.31 & 0.00 \\
\hline $81-82$ & 14179 & 0.16 & 0.03 & 16.6 & 0.8 & 153 & 33 & 0.30 & 0.00 \\
\hline $90-91$ & 14821 & 0.38 & 0.07 & 24.8 & 1.1 & 195 & 27 & 0.37 & 0.01 \\
\hline $101-102$ & 15598 & 1.56 & 0.25 & 46.8 & 2.2 & 194 & 42 & 0.56 & 0.09 \\
\hline $109-110$ & 16120 & 0.71 & 0.13 & 24.6 & 1.1 & 193 & 27 & 0.78 & 0.01 \\
\hline $125-126$ & 17109 & 0.31 & 0.05 & 19.6 & 0.9 & 125 & 27 & 0.86 & 0.14 \\
\hline $141-142$ & 17745 & 0.76 & 0.13 & 30.6 & 1.5 & 235 & 53 & 1.21 & 0.20 \\
\hline $161-162$ & 18109 & 0.29 & 0.05 & 27.1 & 1.2 & 118 & 17 & 1.12 & 0.02 \\
\hline $171-172$ & 18291 & 0.48 & 0.08 & 21.1 & 1.0 & 130 & 18 & 1.32 & 0.21 \\
\hline $181-182$ & 19148 & 0.26 & 0.05 & 36.2 & 1.7 & 69 & 15 & 1.28 & 0.02 \\
\hline 191-192 & 20041 & 0.29 & 0.05 & 38.5 & 1.8 & 79 & 11 & 1.44 & 0.24 \\
\hline $205-206$ & 21062 & 0.43 & 0.08 & 25.3 & 1.2 & 85 & 12 & 1.25 & 0.02 \\
\hline $221-222$ & 21829 & 0.50 & 0.10 & 15.0 & 0.7 & 168 & 24 & 1.97 & 0.32 \\
\hline $240-241$ & 22913 & 0.28 & 0.05 & 14.2 & 0.7 & 116 & 16 & 1.50 & 0.02 \\
\hline $261-262$ & 23466 & 0.35 & 0.07 & 16.7 & 0.8 & 79 & 17 & 1.58 & 0.03 \\
\hline $290-291$ & 24739 & 0.52 & 0.09 & 17.7 & 0.8 & 169 & 24 & 1.27 & 0.02 \\
\hline $309-310$ & 25440 & 1.26 & 0.25 & 17.3 & 0.8 & 204 & 46 & 2.92 & 0.50 \\
\hline $330-331$ & 26032 & 0.71 & 0.13 & 7.6 & 0.4 & 273 & 60 & 1.24 & 0.21 \\
\hline $341-342$ & 26402 & 0.66 & 0.12 & 22.7 & 1.1 & 179 & 25 & 1.67 & 0.27 \\
\hline $361-362$ & 27768 & 0.24 & 0.04 & 21.4 & 1.0 & 241 & 34 & 0.74 & 0.01 \\
\hline $371-372$ & 28451 & 0.22 & 0.04 & 12.0 & 0.6 & 80 & 17 & 0.88 & 0.14 \\
\hline $379-380$ & 28998 & 0.44 & 0.08 & 18.0 & 0.8 & 131 & 29 & 0.69 & 0.01 \\
\hline
\end{tabular}

71 *Indicates suspected contamination. 
75 Table S3. TN057-13-4PC organic carbon concentrations. RSD is $3 \%$ based on duplicate analysis of $\sim 10 \%$ of 76 samples.

\begin{tabular}{|c|c|c|}
\hline Depth $(\mathrm{cm})$ & Age (yr) & $\mathrm{C}_{\text {org }}$ (weight $\%$ ) \\
\hline $0-1$ & 18 & $2.29 *$ \\
\hline $10-11$ & 305 & 0.42 \\
\hline $20-21$ & 749 & 0.57 \\
\hline $40-41$ & 1259 & 0.41 \\
\hline $60-61$ & 1769 & 0.27 \\
\hline $80-81$ & 2295 & 0.27 \\
\hline $100-101$ & 2824 & 0.37 \\
\hline $120-121$ & 3353 & 0.29 \\
\hline $140-141$ & 3882 & 0.35 \\
\hline $160-161$ & 4410 & 0.31 \\
\hline $180-181$ & 4939 & 0.31 \\
\hline $200-201$ & 5468 & 0.42 \\
\hline $220-221$ & 5997 & 0.44 \\
\hline $240-241$ & 6526 & 0.49 \\
\hline $260-261$ & 6998 & 0.43 \\
\hline $300-301$ & 7549 & 0.36 \\
\hline $340-341$ & 8100 & 0.41 \\
\hline $380-381$ & 8651 & 0.54 \\
\hline $420-421$ & 9203 & 0.38 \\
\hline $470-471$ & 9631 & 0.36 \\
\hline $510-511$ & 10164 & 0.33 \\
\hline $560-561$ & 10988 & \\
\hline $580-581$ & 11367 & 0.31 \\
\hline $610-611$ & 11936 & 0.30 \\
\hline $640-641$ & 12505 & 0.27 \\
\hline $670-671$ & 13073 & 0.37 \\
\hline $700-701$ & 13642 & $0.82 *$ \\
\hline $730-731$ & 14211 & 0.38 \\
\hline $760-761$ & 16163 & 0.47 \\
\hline $770-771$ & 17062 & 0.42 \\
\hline $780-781$ & 17960 & 0.43 \\
\hline $790-791$ & 18859 & 0.56 \\
\hline $800-801$ & 19758 & 0.60 \\
\hline $810-811$ & 20657 & 0.46 \\
\hline $820-821$ & 21556 & 0.38 \\
\hline $830-831$ & 22454 & 0.37 \\
\hline $840-841$ & 23353 & 0.39 \\
\hline $850-851$ & 24252 & 0.34 \\
\hline $860-861$ & 25151 & 0.35 \\
\hline
\end{tabular}




\begin{tabular}{lll}
$870-871$ & 26050 & 0.37 \\
$880-881$ & 26949 & 0.44 \\
$890-891$ & 27847 & 0.48 \\
$900-901$ & 28746 & 0.47 \\
$910-911$ & 29645 & 0.46 \\
$920-911$ & 30604 & 0.38 \\
\hline
\end{tabular}

77 *Indicates suspect measurement 
78 Table S4. RC13-254 organic carbon concentrations. RSD is 3\% based on duplicate analysis of $\sim 10 \%$ of samples.

\begin{tabular}{ccc}
\hline Depth (cm) & Age (yr) & $\mathrm{C}_{\text {org }}$ (weight \%) \\
\hline $0-1$ & 3500 & 1.06 \\
$5-6$ & 4625 & 0.96 \\
$10-11$ & 5750 & 0.66 \\
$15-16$ & 6950 & 0.29 \\
$20-21$ & 8293 & 0.35 \\
$25-26$ & 9100 & 0.30 \\
$30-31$ & 10100 & 0.26 \\
$35-36$ & 11050 & 0.33 \\
$41-42$ & 11650 & 0.36 \\
$50-51$ & 12393 & 0.34 \\
$61-62$ & 13125 & 0.34 \\
$70-71$ & 13575 & 0.36 \\
$81-82$ & 14179 & 0.37 \\
$90-91$ & 14821 & 0.62 \\
$101-102$ & 15598 & 0.62 \\
$109-110$ & 16120 & 0.57 \\
$125-126$ & 17109 & 0.55 \\
$141-142$ & 17745 & 0.82 \\
$161-162$ & 18109 & 0.58 \\
$171-172$ & 18291 & 0.59 \\
$181-182$ & 19148 & 0.57 \\
$191-192$ & 20041 & 0.61 \\
$205-206$ & 21062 & 0.60 \\
$221-222$ & 21829 & 0.66 \\
$240-241$ & 22913 & 0.48 \\
$261-262$ & 23466 & 0.53 \\
$290-291$ & 24739 & 0.66 \\
$309-310$ & 25440 & 0.63 \\
$330-331$ & 26032 & 0.55 \\
$341-342$ & 26402 & 0.54 \\
$361-362$ & 27768 & 0.54 \\
$371-372$ & 28451 & 0.43 \\
$379-380$ & & 0.59 \\
\hline & 2898 & \\
\hline & & \\
\hline
\end{tabular}

79

80

81 
82 Table S5. xs ${ }^{230} \mathrm{Th}_{0}$ values for RC13-254 (Kumar, 1994).

\begin{tabular}{ccc}
\hline Depth $(\mathrm{cm})$ & $\mathrm{xs}^{230} \mathrm{Th}_{0}(\mathrm{dpm} / \mathrm{g})$ & $\begin{array}{c}{ }_{\mathrm{x}}{ }^{230} \mathrm{Th}_{0} 1 \sigma \\
\text { uncertainty }(\mathrm{dpm} / \mathrm{g})\end{array}$ \\
\hline 13 & 10.34 & 0.175 \\
18 & 9.40 & 0.157 \\
34 & 7.74 & 0.167 \\
123 & 3.06 & 0.096 \\
172 & 2.46 & 0.071 \\
432 & 4.66 & 0.151 \\
\hline
\end{tabular}

83

84

85 
87 Anderson, R. F., Ali, S., Bradtmiller, L. I., Nielsen, S. H. H., Fleisher, M. Q., Anderson, B. E.,

88 and Burckle, L. H.: Wind-driven upwelling in the Southern Ocean and the deglacial rise in

89 atmospheric $\mathrm{CO}_{2}$, Science, 323, 1443-1448, 2009.

90 Bradtmiller, L. I., Anderson, R. F., Fleisher, M. Q., and Burckle, L. H.: Comparing glacial and

91 Holocene opal fluxes in the Pacific sector of the Southern Ocean. In: Paleoceanography,

$92 \quad$ PA2214, 2009.

93 Chase, Z., Anderson, R. F., Fleisher, M. Q., and Kubik, P. W.: Accumulation of biogenic and

94 lithogenic material in the Pacific sector of the Southern Ocean during the past 40,000 years,

95 Deep-Sea Research II, 50, 799-832, 2003.

96 Dezileau, L., Bareille, G., Reyss, J. L., and Lemoine, F.: Evidence for strong sediment

97 redistribution by bottom currents along the southeast Indian ridge, Deep-Sea Research I, 47,

$98 \quad 1899-1936,2000$.

99 Francois, R., Bacon, M. P., Altabet, M. A., and Labeyrie, L. D.: Glacial/interglacial changes in 100 sediment rain rate in the SW Indian sector of Subantarctic waters as recorded by ${ }^{230} \mathrm{Th},{ }^{231} \mathrm{~Pa}, \mathrm{U}$, 101 and $\delta^{15} \mathrm{~N}$, Paleoceanography, 8, 611-629, 1993.

102 Francois, R., Frank, M., van der Loeff, M. M. R., and Bacon, M. P.: Th-230 normalization: An 103 essential tool for interpreting sedimentary fluxes during the late Quaternary. In: 104 Paleoceanography, PA1018, 2004.

105 Kumar, N.: Trace metals and natural radionuclides as tracers of ocean productivity, Ph.D. Ph.D., 106 Columbia University, 336 pp., 1994. 
107 Kumar, N., Anderson, R. F., Mortlock, R. A., Froelich, P. N., Kubik, P., Dittrichhannen, B., and 108 Suter, M.: Increased biological productivity and export production in the glacial Southern Ocean, 109 Nature, 378, 675-680, 1995.

110 Skinner, L. C., Fallon, S., Waelbroeck, C., Michel, E., and Barker, S.: Ventilation of the deep 111 Southern Ocean and deglacial $\mathrm{CO}_{2}$ rise, Science, 328, 1147-1151, 2010.

112 Skinner, L. C., Waelbroeck, C., Scrivner, A. E., and Fallon, S. J.: Radiocarbon evidence for 113 alternating northern and southern sources of ventilation of the deep Atlantic carbon pool during 114 the last deglaciation, Proceedings of the National Academy of Sciences of the United States of 115 America, 111, 5480-5484, 2014.

116 


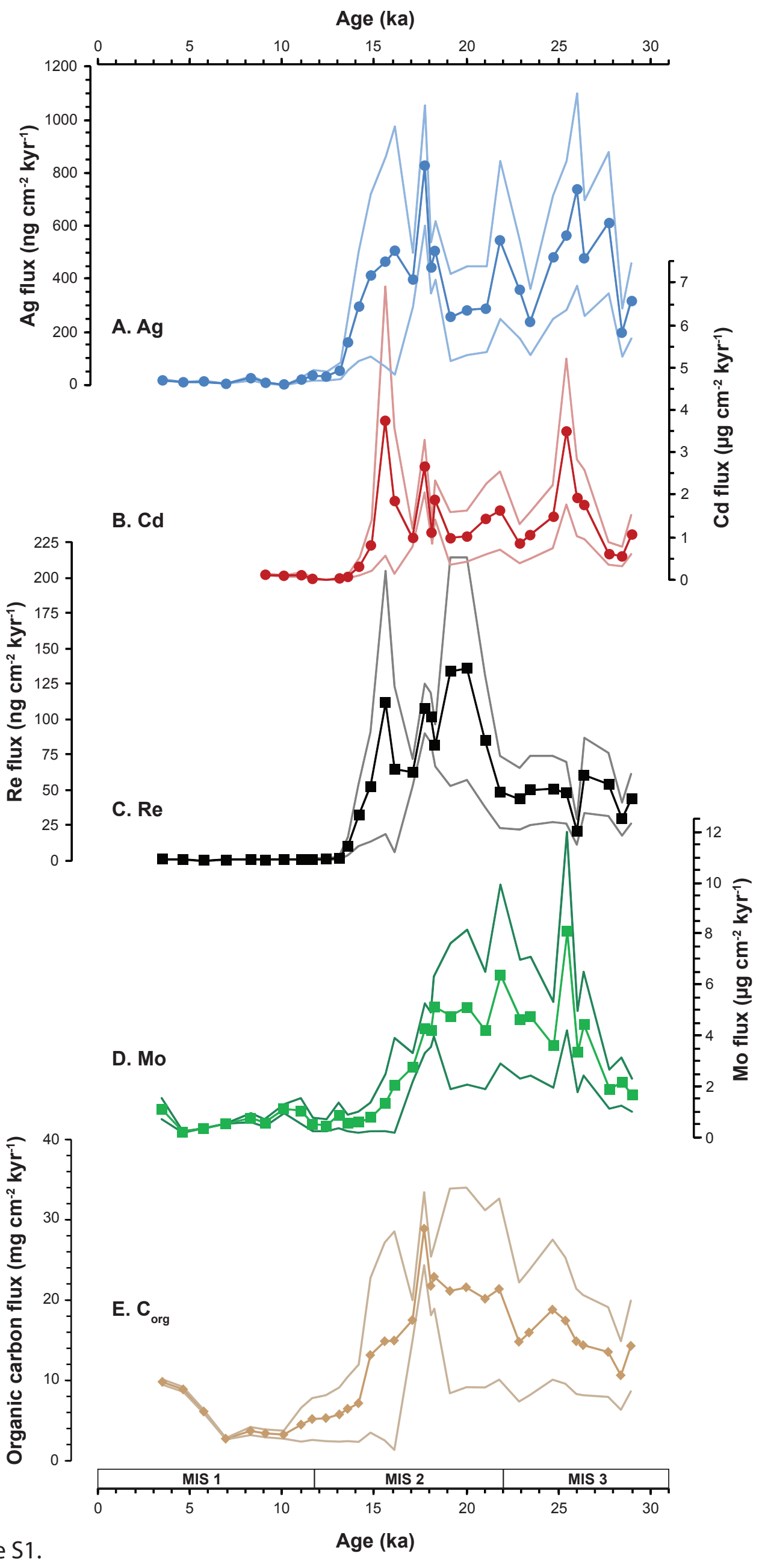

Figure S1. 


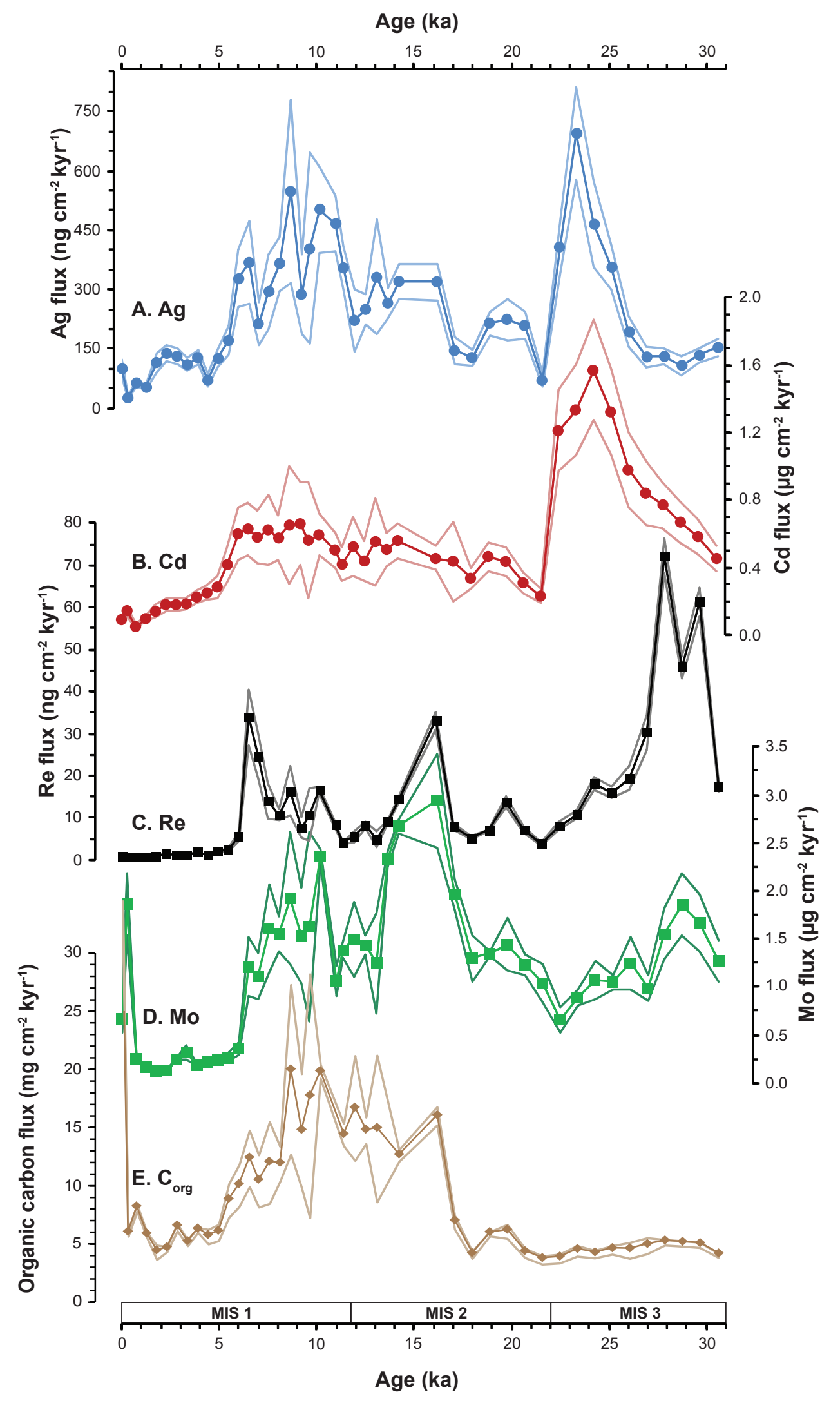

Figure S2. 